traits, which do not readily fit with the others (attention deficit hyperactivity disorder (ADHD) and learning disabilities). A third problem is that schizoaffective disorder was included among the bipolar spectrum disorders in the analyses, a decision that requires further justification.

A fourth problem is that, as described in a previous article, ${ }^{2}$ a diagnosis of bipolar affective disorder not otherwise specified was given to participants who presented with manic symptoms meeting threshold DSM-IV diagnostic criteria but not minimal duration criteria. It is possible that this was the reason for a statistically significant difference in the cumulative incidence of bipolar spectrum disorders between the offspring of well parents and the offspring of parents with a bipolar disorder. Finally, 23\% of participants in the group of offspring of a parent with bipolar disorder 1 were recruited within families, making it unclear how many participants had a parent who did not have the disorder.

1 Duffy A, Horrocks J, Doucette S, Keown-Stoneman C, McCloskey S, Grof P. The developmental trajectory of bipolar disorder. Br J Psychiatry 2014; 204: $122-28$.

2 Duffy A, Alda M, Crawford L, Milin R, Grof P. They early manifestations of bipolar disorder: a longitudinal prospective study of the offspring of bipolar parents. Bipolar Disord 2007; 9: 828-38.

Marie-Pierre Chenard-Poirier, MD, Joel Paris, MD, Psychiatry Department, McGill University, Canada. Email: marie-pierre.chenard-poirier@mail.mcgill.ca

doi: 10.1192/bjp.205.2.158b

Authors' reply: The clinical staging model proposed represents an aggregate view based on results from an ongoing, prospective study of a unique, high-risk cohort. In prior analyses, we found evidence that ADHD and other childhood neurodevelopmental presentations occurred at a higher unadjusted rate in the offspring of parents with lithium-non-responsive illness compared with the offspring of parents with lithium-responsive illness. ${ }^{1,2}$ In this updated analysis, instead of unadjusted lifetime rates we used cumulative incidence, which takes into account censoring and variable age at last assessment and Cox proportional hazard models adjusted for sibling correlation, gender and socioeconomic status. With longer observation, the unadjusted rate of psychotic disorders is now significantly elevated in the offspring of parents with lithium-non-responsive illness compared with the offspring of parents with lithium-responsive illness.

Second, cluster A traits and cognitive deficits are known antecedents to psychotic disorders and therefore we argue that these do in fact 'fit' with ADHD and learning disabilities as early risk syndromes in this high-risk population. ${ }^{3}$ Third, schizoaffective disorder was included as an end-stage illness in this analysis given the overlap between schizoaffective and psychotic bipolar disorders. ${ }^{4}$ Fourth, all offspring (control and high-risk) were assessed in the same way and all assessments were reviewed masked to family affiliation and diagnoses made by consensus using the same criteria. Therefore, the difference in rates of bipolar disorder not otherwise specified or any other diagnosis cannot be explained by modified diagnostic criteria for high-risk offspring as speculated by Chenard-Poirier \& Paris.

Finally, given the high heritability and estimated likelihood that recurrent major depression in these families reflects the bipolar diathesis, ${ }^{5}$ we expanded recruitment to include the offspring of parents who were siblings of the original bipolar proband and who themselves met lifetime criteria for bipolar disorder or recurrent major depression $(n=20)$. Therefore, every high-risk offspring had one parent with a bipolar or bipolar-related recurrent major depressive disorder. We thank Chenard-Poirier \& Paris for raising these points and the Journal for allowing us to provide this clarification.
1 Duffy A, Alda M, Hajek T, Sherry SB, Grof P. Early stages in the development of bipolar disorder. J Affect Disord 2010; 121: 127-35.

2 Duffy A, Alda M, Crawford L, Milin R, Grof P. The early manifestations of bipolar disorder: a longitudinal prospective study of the offspring of bipolar parents. Bipolar Disord 2007; 9: 828-38.

3 Murray RM, Sham P, Van Os J, Zanelli J, Cannon M, McDonald C. A developmental model for similarities and dissimilarities between schizophrenia and bipolar disorder. Schizophr Res 2004; 71: 405-16.

4 Pearlson GD, Ford JM. Distinguishing between schizophrenia and other psychotic disorders. Schizophr Bull 2014; 40: 501-3.

5 Blacker D, Lavori PW, Faraone SV, Tsuang MT. Unipolar relatives in bipolar pedigrees: a search for indicators of underlying bipolarity. Am J Med Genet 1993; 48: 192-9.

Anne Duffy, Department of Psychiatry, University of Calgary, Canada. Email: acduffy@ucalgary.ca; Julie Horrocks, Charles Keown-Stoneman, Department of Mathematics and Statistics, University of Guelph, Canada; Sarah Doucette Department of Community Health and Epidemiology, Dalhousie University, Canada; Paul Grof, Mood Disorders Centre of Ottawa and Department of Psychiatry, University of Toronto, Canada.

doi: 10.1192/bjp.205.2.159

\section{An oversimplification of psychosis, its treatment, and its outcomes?}

Jauhar et al's meta-analysis ${ }^{1}$ of randomised controlled trials in cognitive-behavioural therapy for psychosis (CBTp) is broadly consistent with previous results: ${ }^{2}$ that is, there is an overall significant but modest impact on psychotic symptoms, with blinded studies showing lower effect sizes than those that are not blinded. However, there are a number of problems with this study and especially with its conclusions.

Jauhar et al conclude that they find the advocacy by government (including NICE) for CBTp 'puzzling', bearing in mind the low effect sizes found for psychotic symptoms. However, I find it puzzling that the authors comment on NICE recommendations, since a third of the studies included for their overall symptoms analysis (12/34) were not based on therapies recommended by NICE in the first place (based on what we know is effective from the literature so far): they were either group or brief CBT studies. Three further studies were in Chinese, so their relevance to NICE recommendations is hard to tell.

It is a testament to the far-reaching effects of CBTp that the analyses revealed any effects at all, since the authors looked at outcomes that were not always targeted by the therapy. For instance, only a few of the 34 studies included for negative symptoms actually targeted such symptoms specifically. Furthermore, severity of positive symptoms/hallucinations was used as the outcome for studies that did not hypothesise changes in psychotic symptoms since the target was on compliance with command hallucinations, ${ }^{3}$ emotional dysfunction, ${ }^{4}$ or social functioning. ${ }^{5}$ By contrast, outcomes on depression, anxiety or distress as a result of psychotic symptoms, and trials targeting self-esteem, post-traumatic symptoms, suicidality, or substance misuse, which are all main and legitimate targets in CBTp, were excluded.

The criteria for studies to be included in the final analyses were idiosyncratic. Perhaps the most surprising was the decision to exclude studies that targeted hallucinations specifically from their positive symptoms analyses. A separate 'supplementary' meta-analysis was carried out for those studies, with an effect size of 0.34 , which is not reported in the abstract (where only the lower -0.25 effect on positive symptoms is reported). Clinicians familiar with clinical presentations of patients with psychosis might be surprised at their rationale for excluding trials because patients had a dual diagnosis, or had medication-resistant psychotic symptoms but no further diagnosis specification. None of the follow-up data available was included, meaning that the 